\title{
Editorial: Alkali-activated materials, geopolymers, concrete and sustainability
}

\author{
John L. Provis \\ Department of Materials Science \& Engineering, University of Sheffield, \\ Sheffield, UK
}

Alkali activation is one of the most active, and hotly debated, topics in research and innovation in the field of cement and concrete at present. The use of alkaline activators to accelerate reaction and strength development has been proposed as a method by which low-value waste or geological materials can be converted into cementitious binders for use in the production of concretes with desirable performance and durability, and is now being demonstrated and applied at full production scale in several parts of the world. The number of research publications in this area is growing rapidly, and the level of understanding of the fundamental chemistry, materials science and engineering properties of this class of materials is improving as new techniques and expertise are brought to bear on the questions at hand. This is continuing to enhance and underpin efforts in commercialisation and standardisation of alkaliactivated materials for application in concrete production at industrial scale.

However, there are a number of points related to these materials that still require further insight and investigation, and so the main purpose of this editorial is to highlight some such areas that are of relevance to the readership of Magazine of Concrete Research.

First and foremost, the large-scale deployment of any novel type of concrete requires that specifiers and end-users have faith in the long-term performance of the material while in service. This requires the following.

- That the binder itself is stable (chemically and dimensionally)

- the combination of binder and aggregates does not cause problems

- the embedded steel reinforcing is able to be protected from corrosion for an appropriate length of time.

Each of these characteristics can only truly be proven through field testing of the performance of the material in service. However, because the potential degradation mechanisms are usually slow and the required service life is very long, accelerated laboratory testing is applied to provide confidence in the performance of a material. There is an extensive catalogue of standard tests that have been demonstrated to provide a good prediction of field performance for concretes based on Portland cement, and so it is often assumed that when an alternative material is tested according to the same protocols, the test results will provide a similarly accurate prediction of in-service performance. For the case of alkali-activated materials, whose chemistry is in many ways very different from that of Portland cement, this assumption requires validation, and this is the focus of intensive international research efforts (including the work of RILEM Technical Committee 247DTA) at present. This is an area in which the readership of the Magazine of Concrete Research is certainly well-positioned to provide valuable information to the community in general, as the most important validation required is related to tests that are conducted on full-scale concrete elements.

The validation of concrete performance testing, and the correlation between laboratory test results and field performance is particularly critical in the standardisation of alkaliactivated materials. There are ongoing efforts in various jurisdictions focused on performance-based standardisation of alkali-activated cements and concretes; one such example is in the UK where a 'publicly available specification' (PAS) for this class of materials is currently being prepared for publication in 2016. This specification is a pre-standardisation step in the British Standards Institution process, which is sponsored by interested parties, drafted by technical authors, and revised and approved by an industry steering group via public consultation, and is based on a performance-testing approach to the prediction of in-service durability of the alkaliactivated concretes. Probably the main challenge in drafting a document such as this, for a class of materials that is as diverse as alkali-activated materials, is ensuring that the consumer and end-user are fully protected, while enabling the producers to innovate and provide as broad as possible a range of materials and formulations. The use of accelerated laboratory testing coupled with field trials for validation, rather than prescribing formulations or compositions, leaves a vast scope for innovative solutions to the design of alkali-activated concrete. 
However, to underpin efforts such as this, a deep and detailed science base is required. There must be a mature understanding of the processes and mechanisms (both physical and chemical) by which degradation of the concretes can take place. Due to the way in which alkali-activation technology has evolved, largely starting from chemical-level studies involving paste mixes, followed by scale-up to mortar and concrete production, the open literature currently demonstrates a much better understanding of the chemical (chloride, carbonation, sulfate, alkali-silica) than the physical (freeze-thaw, creep, shrinkage) processes that can cause damage to alkali-activated concretes. This is a situation that will certainly evolve as more concretefocused researchers and practitioners become active in publishing the results of larger-scale studies, and these materials truly move beyond the laboratory and into large-scale application. The current rapid growth in commercial-scale alkali-activated concrete production, particularly in Australia where major infrastructure works (including road projects and an airport) now feature alkali-activated concretes in prominent and responsible roles, indicate that there is a high degree of enduser confidence in these materials.

This, therefore, signposts some important areas in which researchers can genuinely contribute to, and generate impact from, the deployment of alkali-activated materials. Any evidence that can underpin the relationship between laboratory testing and field performance, enabling the prediction of the long-term behaviour of alkali-activated concretes under various environmental and mechanical loads, will be of significant value. However, to gain full benefit from such testing programmes, it is required to move beyond the analysis of a single mix design, or even a single source of precursor materials, and to provide information and trends that are generically applicable across at least a section of the diversity of materials that can be used as precursors to produce alkali-activated materials. It is certain that valuable information can nonetheless be gained from a study involving a single source of materials, but the ability to identify generic trends across a class of materials is central to the ability to truly predict material performance in service.

From this viewpoint, it is clear that this rapidly evolving research area still poses many fundamental questions that require answers, to enable and accelerate the uptake of alkali activation as one possible route to environmentally responsible concrete production. This should remain an area of high research activity for many years to come, and thus raises interesting and important questions for the readership of the Magazine of Concrete Research. 